\title{
AUTONOMOUS CONTROL OF HYDRAULIC MOBILE APPLICATIONS - A 21-TON EXCAVATOR CASE STUDY
}

\author{
Dr. Tim Opperwall*, Ben Holter, Simon Yardley \\ Husco International, 2239 Pewaukee Rd, Waukesha, WI 53188, USA \\ * Corresponding author: Tel.: +1 262 513-4266; E-mail address: timothy.opperwall@husco.com
}

\begin{abstract}
Automation of mobile construction and agricultural equipment has gained wide acceptance based on increases in productivity, safety, and precision; while also helping upskill operators. On construction equipment, after-market automation of earthmoving crawler dozers and graders has driven a conversion of machines to electro-hydraulic (EH) implement control and integration into digital worksites. Unlike the aforementioned machines, conversion of the excavator into a semi or fully autonomous machine presents significant challenges due to kinematics, variable loads, non-linear multi-function of implements, safety, and robustness. The present work demonstrates the retrofit of a pilot-operated 21ton excavator and development of automated controls to address these challenges. The operator pilot joysticks and existing hydraulic system were retained, while adding capability for autonomous functionality with integrated hardware, controls, and kinematic solvers within a production viable environment. Autonomous features for path planning, multi-function actuator velocity control, EH controls, and safety were developed to prove the value of precise and low latency control hardware for EH excavator operation.
\end{abstract}

Keywords: Hydraulic mobile applications, Electro-Hydraulics, Autonomous Excavator, Smart construction sites

\section{INTRODUCTION}

The present work describes a technically proven and commercially viable system to provide digital control and autonomous implement position control of a standard pilot operated machine. A 21-ton excavator was chosen as the applied research vehicle as it provided significant inherent challenges and is typically the first prime mover on construction sites globally. Some of the key challenges such as balancing the pressure, power, and flow of multiple functions in relation to command and desired function path are included in the research.

The majority of original equipment manufacturer (OEM) excavators in use today have hydraulic pilot joystick signals controlling the hydraulic main control valve (MCV). Although there are a small number of electrohydraulic (EH) controlled machines emerging on the market as factory fit, few companies have been able to create a system that can easily be retrofitted into an existing circuit while providing precise digital control and enabling of autonomous functions such as auto-grade and geo-fencing. This gap in the market is due to the difficult transform of operator controls to precise kinematic motion. Excavators are different in control strategy than other precision equipment like bulldozers and motor graders in that excavators must coordinate many functions simultaneously at high velocity to control a precise implement position and path. Other types of machines such as crawler dozers and graders must only make small, single function positional adjustments as they move over the earth. This difference has made automated control very difficult from a hardware and software standpoint, which is why the market has lagged on adoption of autonomous excavator control.

Complex construction machines require skilled labour to operate them safely and productively to maximise the significant capital investment of the machine. Bringing in autonomous or augmented functions to support the operator brings several benefits. Automatic grade control can enable a machine to precisely follow a pre-set path or trajectory, minimising 
wasteful under or over cutting of material, speeding up the digging process, and reducing the cognitive load of the operator allowing them to concentrate on other functions. Autonomous and augmented functions can also bring in advanced safety features such as object avoidance to automatically prevent collisions with dangerous, unseen items such as gas infrastructure and overhead powerlines. The only viable option to enable these features is a migration to $\mathrm{EH}$ control of the main control valve. The present work describes a system for converting any typical pilot-controlled machine into an EH controlled machine, while retaining the feel and controls of the original system. Layered on top of that work is demonstration of the EH capability of a retrofitted system through position and velocity control using the existing pumps and control valves on the excavator. The main work under consideration for this research was demonstrating the controls on the hydraulic excavator application on a rented Case CX210 excavator with 4000 operating hours. The pilot pressure control, function velocity control, and path planning controls were developed to work alongside the existing negative control hydraulic system on the machine.

\section{ELECTROHYDRAULIC CONTROLS}

There are three main styles of operator control of hydraulic systems on mobile applications. Namely, manual control, pilot pressure control, and electro-hydraulic (EH) control. Typically, manual controls are used on the smallest and/or most cost sensitive applications. Pilot pressure control is the industry standard and widely accepted for all working machines based on the balance of feel, control, and cost. Electrohydraulic control is gaining acceptance on the market but has traditionally faced headwinds and scepticism with respect to cost, reliability, precision, and responsiveness on excavators.

In comparison to agriculture and other large industries, the construction industry has not achieved the same increases in productivity over the past decades as reported by McKinsey [1]. In part this is due to fragmentation of the market, the high customization and complexity of equipment and job sites, and underinvestment in innovation. One of the largest potential areas to boost productivity is electrification of equipment to enable automation [1]. Smart construction sites have accepted new technologies and increased demand for high precision work. This has driven a rapid transformation in the motor grader and dozer market towards full $\mathrm{EH}$ valves where the operator oversees steering and speed while global positioning systems replace and supplement traditional implement control and base surveying methods.

\subsection{Challenges}

A strong motivation for this paper is to demonstrate the effectiveness of $\mathrm{EH}$ control on excavators through a retrofit of an existing pilot operated system and development of coupled electrical, hydraulic, and kinematic controls. The goal of the research was to demonstrate the effectiveness of the Husco Exacto pilot pressure control system [2] by designing a generic and extensible control system for commanding an excavator in multi-function autonomous operation. Conversion of the excavator into a semi or fully autonomous machine presents significant challenges due to kinematics, variable loads, non-linear multi-function of implements, safety, and robustness. The main deliverables were enhanced performance of the existing hydraulic circuit, and a full speed automated flat grade at $\pm 25 \mathrm{~mm}$ tolerance using the full working range of the machine.

The requirement for the grade to be achieved at full machine speed is to prove that EH controls are not a limiting productivity or precision factor. Operating at the limit of machine performance increases the complexity of the control system when the pump pressure, flow, or power limit are reached as in Figure 1 showing real machine data for a two pump excavator driven by an experienced operator. Flow and power are shared by all the moving functions which is a complexity an operator must compensate for, and must be replaced by sensors and algorithms.

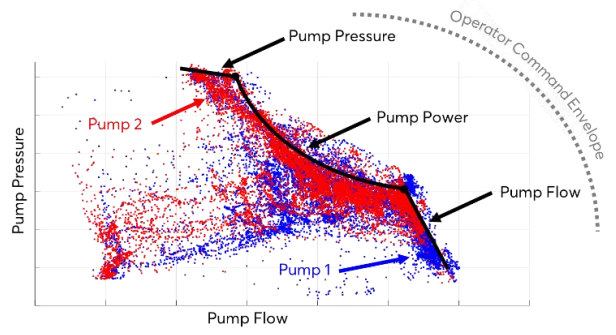

Figure 1: Working excavator power limits. 
These challenges were evaluated with the Husco Exacto system in Figure 2. Exacto is an EH manifold valve with integrated controller, software, sensors and electrical harness. The system includes an advanced current servo with patented algorithms for dealing with changing environment and operating conditions, patent pending closed loop pilot pressure controls with two sets of pressure sensors per implement. The system is designed to interface with surveying/guidance systems and/or OEM instructions over CAN, and can be installed into existing pilot control systems without compromising any existing operator commanded performance or feel.

The Exacto system was also extended to include Husco developed path planning and function velocity control algorithms for this research. To supplement the EH controls, a human machine interface (HMI) was developed for triggering the system and selecting the commanded operation. String potentiometers and inertial measurement units (IMU) were added to facilitate the position and velocity control. This configuration is what was tested in the paper.

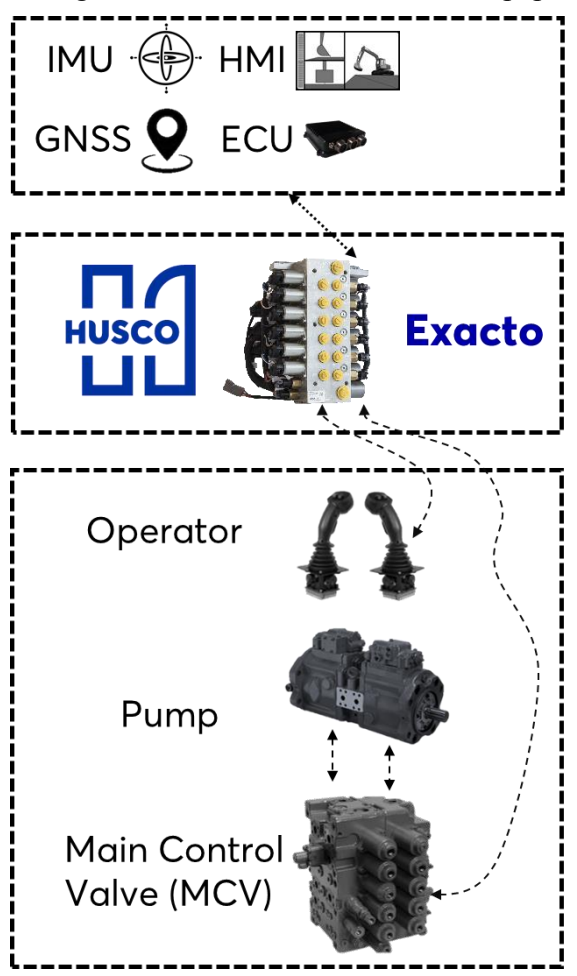

Figure 2: Exacto system implementation.

\subsection{Pilot pressure control}

Electrohydraulic control on the Exacto system is accomplished through the Firepower 2 family of Husco EH solenoid cartridge valves. The main working element is the electrohydraulic proportional relief valve (EPRV).

There are two main options for factory or retrofit using the Exacto system for EH pilot pressure control. The first version which was tested in the present work is a pilot interrupter system that sits between standard pilot joysticks and the spool end caps of the main control valve. When de-energized, the pilot interrupter system operates in pass-through mode where operator pilot joystick pressures reach the $\mathrm{MCV}$ unimpeded; retaining the machine in its original operating mode and operator HMI (the pilot joysticks). When the system is energized, the pilot joysticks can be blocked off to allow full $\mathrm{EH}$ operation or overriding/augmentation of operator commands. The second version allows for retrofit of the machine with digital or analog joysticks and removal of pilot hoses from the machine cab. Both options contain sensors to detect the hydraulic command generated to move the MCV spool and method for monitoring the operator joystick command.

The Firepower 2 EPRVs used by the system are fast, precise, and stable. Closed loop pressure controls leverage knowledge of the system to achieve the highest level of speed and repeatability. Safety and reliability is added to the EH system through monitoring of EPRV performance, detection of system fault or failure, and protection against change in $\mathrm{EH}$ performance over the life of the parts.

\subsection{Velocity control}

The development of the multi-function actuator velocity control system leveraged existing knowledge of hydraulic control systems and applied it to the reference machine. There is significant variation in excavator working function performance depending on the architecture of the hydraulic system, the loading of the individual functions, and machine to machine production variation. To establish multifunction harmony and control of the machine for coordinated high-speed movement, a plant model of the machine was developed with no prior knowledge of the valve design or flow 
prioritization present on the existing negative control system on the CX210 excavator. No modifications were made to the existing hydraulic architecture other than installing the Exacto system in between the MCV and joystick pilot control valves. The multi-function system calculates the required feed-forward pilot pressures to achieve the commanded ratio of multi-function cylinder velocity commands. A closed loop velocity control system was wrapped around the system model to correct both instantaneous and long term errors.

\subsection{Position control}

The main focus of the research was development of the position control system included point inputs, path planning, cost functions, and coordination with the velocity control and feedbacks. There has been significant academic and industry work [3][4] on the topic of path planning and coordinated movement in multiple actuators. Hydraulic system instabilities, latencies, and load dependence increase the difficulty in creating a stable control system.

For reference, in a three-function excavator system (typically named boom, arm, and bucket), there are three degrees of freedom which combine to achieve the desired motion of the end effector. In the selected reference case, the controlled outputs included the position of the tip of the bucket in a cartesian x-y coordinate space, and an angle of attack of the bucket denoted by ' $a$ '. For an example trajectory, the contributions from each function to the bucket tip position are shown in Figure 3. Each function pivot in a working arc centered on the respective pin. Each contribution can be calculated by solving the system of equations for the required function positions to achieve a specific location or the tip of the bucket and desired bucket angle over time.

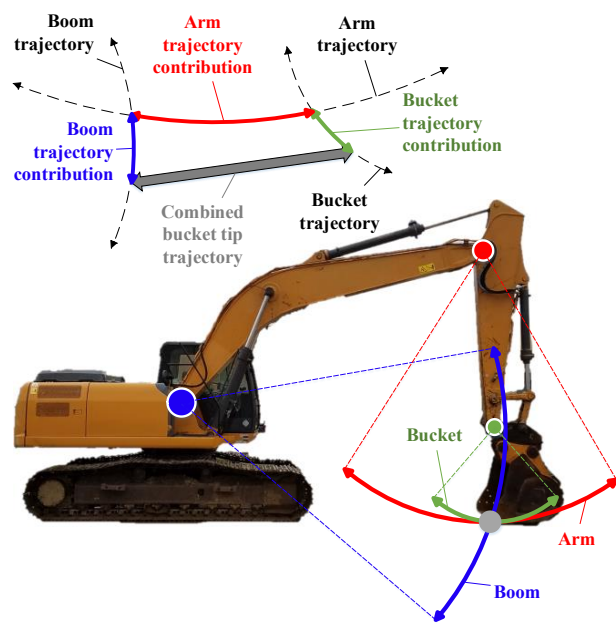

Figure 3: Excavator kinematics.

A set of path points was used to determine a path surface and bucket angle of attack. These points can be inserted into the controls via the HMI or computer connection.

The excavator contains many non-linear behaviors, so stable handling of induced errors and latencies is a key part of the control algorithm. An extrapolation of the trajectory was calculated from positional and velocity feedbacks as shown in Figure 4. Based on the proximity to the desired path, a blending of the perpendicular and following vectors was combined for both the current and projected positions.

The logic and flow of the algorithm can be seen in Figure 5. Positional and velocity feedbacks are combined with a kinematic model of the machine to understand the location of the bucket tip and angle of attack. The vector of travel is used to project a vector of travel for both the current moment in time and the future projected value as shown in Figure 4. Based on an input set of target coordinates, the projected

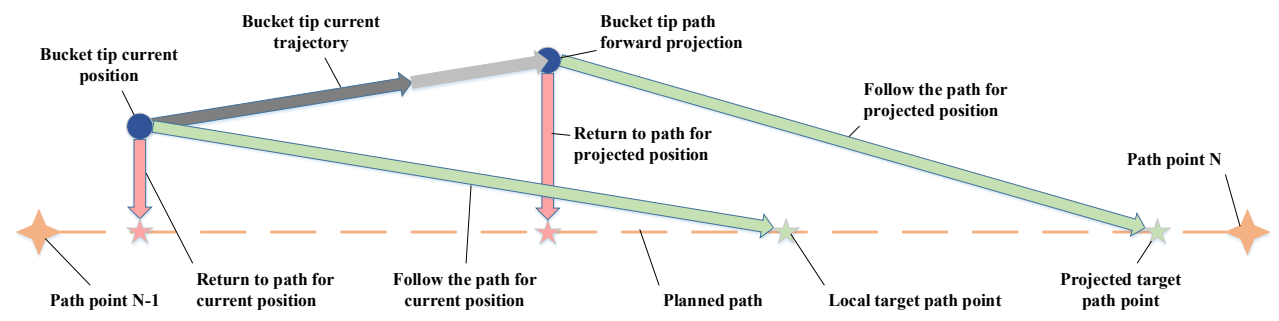

Figure 4: Path planning algorithm 


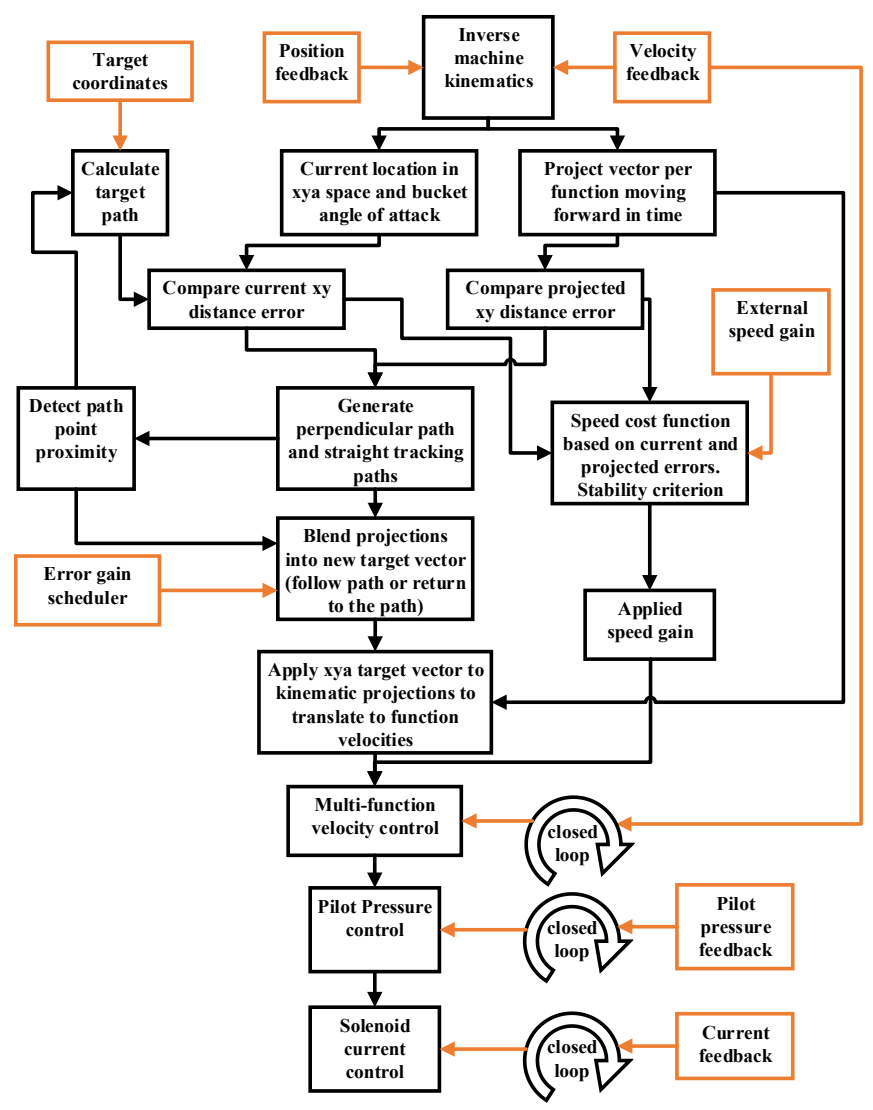

Figure 5: Path planning algorithm details and machine control architecture.

vector of travel is compared to the targeted path surface to generate a set of error terms. Additional error terms are generated based on system stability and acceleration. These error terms are combined into cost functions to govern the allowable speed of the system and change of states.

The cost functions and projected vectors of travel are blended to create a new target command vector, which can then be transformed into new function velocity commands. Closed loop velocity control of all function actuators is then completed iteratively. Each transient velocity control loop generates a set of pilot differential pressures for each control spool within the MCV in order to accomplish the desired velocity of the actuator. The core Exacto EH closed loop control then generates the desired pilot pressure differential using EPRVs.

\section{VALIDATION AND RESULTS}

The described system was designed, installed and tuned on the machine. With proper hardware, the hardware installation can be accomplished in less than one day with one technician. The installation is shown in Figure 6. The headline results demonstrate a system that successfully achieved a full automated grade using three functions (boom, arm, \& bucket) at $95 \%$ of the flow capacity from the machine for the required motion and at a precision of $\pm 25 \mathrm{~mm}$ of the targeted grade. The maximum capacity of the machine was benchmarked by both time for cylinder stroke and pilot pressure commanded relative to maximum pilot pressure. 


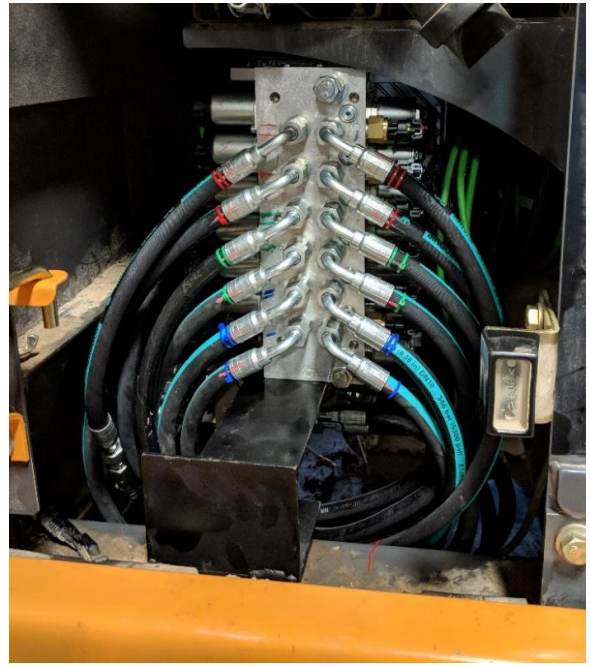

Figure 6: Installation of Exacto pilot interrupter

\subsection{Pilot pressure results}

The step response of the pilot pressure control system was benchmarked against the baseline machine. The EH system is used to replicate the operator command in a control-by-wire manner. The EH pilot control system has been proven to achieve response to $>10 \mathrm{~Hz}$ before reaching a $3 \mathrm{~dB}$ down value as installed on a machine. The spool response can be faster in EH mode than the original pilot controlled system since the EPRV with advanced algorithms can deliver a faster response and higher flow rate than the pilot joysticks, as well as shaping of commands to avoid overshoots. This response is shown in Figure 7 comparing the Exacto pilot pressure response characteristic to a conventional mobile hydraulic solution and the machine response. As part of the study, the machine natural frequencies were also benchmarked.

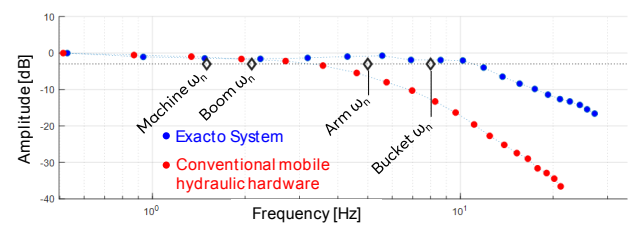

Figure 7: Pilot pressure step response.

The results demonstrated that the core Exacto EH system is not limiting the performance of the machine and can deliver the control efforts necessary to reach the full responsiveness of the function actuators.

\subsection{Position and velocity control results}

Establishing the full working range of the machine for a grading operation began with setting the bucket angle of attack to the desired angle and placing the bucket near the grading surface at maximum reach of the boom and arm. An example full range autonomous grade is shown in Figure 8. This includes the arm crossing beneath its pivot point while maintaining stable control as the load and boom motion switches directions. Figure 8 shows the high speed grade at a precision of $\pm 25 \mathrm{~mm}$ of the targeted surface. The only operator input was the target path points for the start and end of the grade, and an enabling command.

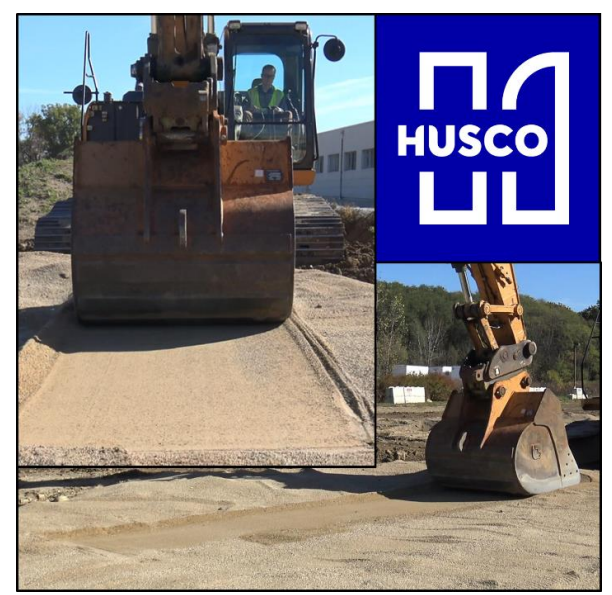

Figure 8: Flat grade demonstration.

The pilot pressure, actuator velocity, and bucket tip position results for the example 7 meter grade over 7.2 seconds is shown in Figure 9. The velocity and position for high difficulty excavator working tasks demonstrates the effectiveness of the algorithms developed for the research vehicle and the EH controls.

An additional interesting test case for path planning and general excavator control algorithms, and one that is very difficult even for a proficient operator, is to draw a circle. The circle requires all functions to work in both extend and retract directions at varying speeds in different quadrants of the circle. A 14 second long exposure of a $2.5 \mathrm{~m}$ diameter circle was taken of the fully automated machine with a light on the bucket tip shown in Figure 10. 

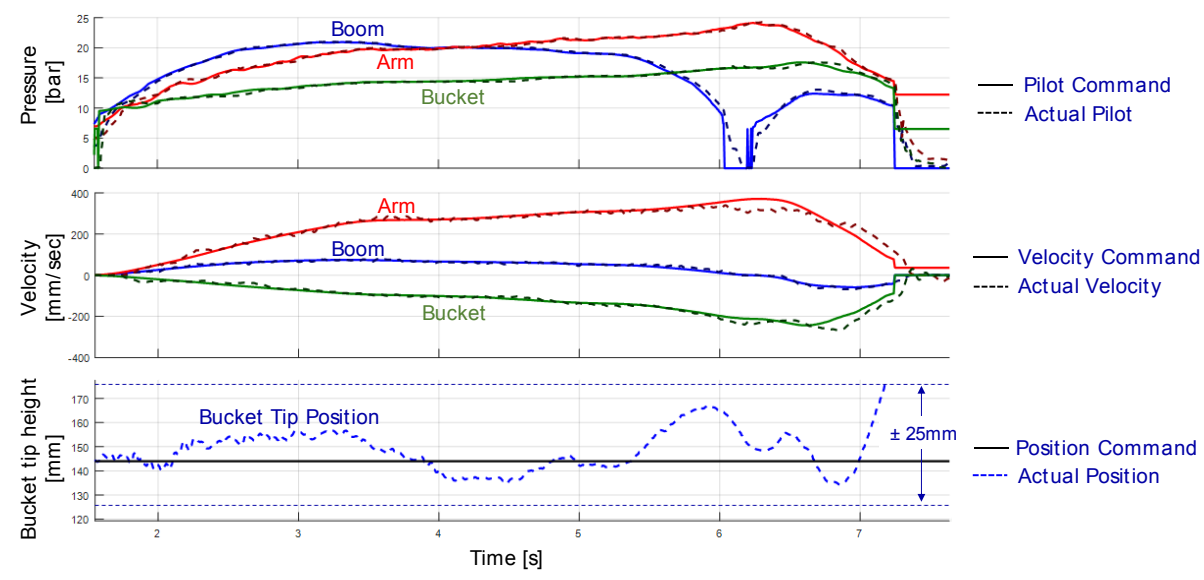

Figure 9: Flat grade results in automatic EH control

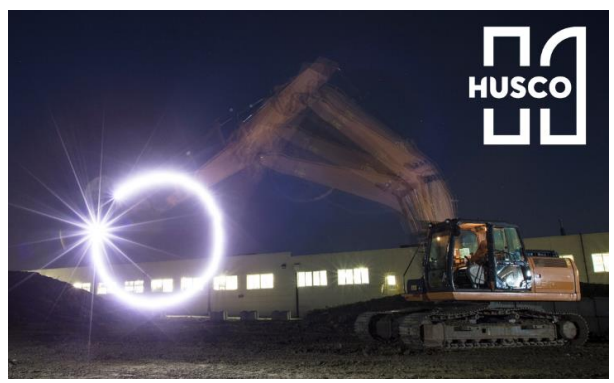

Figure 10: Position control demonstration

\section{NEXT STEPS FOR MACHINE CONTROL}

The present work demonstrated the ease of install of the Exacto pilot interrupter system and many of the benefits of EH control on excavator applications through a compact retrofit pilot manifold assembly.

After developing functional position and velocity control of all actuators, additional capabilities for safety and geo-fencing were explored and can be expanded in future work. With the command-by-wire methods, an expert operator can retain all the feel of a pilot control joystick system while also gaining computer override controls to stop function movement when reaching boundaries set by sensors or environmental hazards. Further development of the system and algorithms would be better integration with IMUs and GNSS systems, development of hardware HMI technology, and expansion of blended operator augmentation methods. Deployment of EH on excavators through both retrofit and factory-fit unlocks the potential for significant productivity improvements to help job sites keep up with global construction demand.

\section{NOMENCLATURE}

$E P R V$ Electrohydraulic proportional relief valve

EH Electro-Hydraulic

$M C V$ Main control valve

$C A N$ Controller area network

HMI Human-machine interface

$I M U$ Inertial measurement unit

$E C U$ Engine control unit

GNSS Global navigational satellite system

\section{REFERENCES}

[1] McKinsey Global Institute (2017), Reinventing construction: a route to higher productivity, https://www.mckinsey.com/industries/capitalprojects-and-infrastructure/ourinsights/reinventing-construction-through-aproductivity-revolution

[2] https://www.husco.com/wpcontent/uploads/2019/11/Exacto-article.-v2.pdf

[3] Gasparetto A, Boscariol P, Lanzutti A, and Vidoni R (2015) Path planning and trajectory planning algorithms: a general overview. Mechanisms and Machine Science, vol. 29

[4] Willms A, Yang S (2006) Real-Time Robot Path Planning via a Distance-Propagating Dynamic System with Obstacle Clearance. IEEE Trans. Syst. vol. 38 